\title{
Value of $\alpha$-fetoprotein as an early biomarker for treatment response to sorafenib therapy in advanced hepatocellular carcinoma
}

\author{
ANA ISABEL PLANO SÁNCHEZ ${ }^{1}$, LUCÍA VELASCO ROCES ${ }^{1}$, ISABEL ZAPICO GARCÍA ${ }^{1}$ EVA LÁZARO LÓPEZ \\ MIGUEL ANGEL CALLEJA HERNANDEZ ${ }^{2}$ MARIA ISABEL BAENA PAREJO ${ }^{2}$ and JAIME PEÑA-DÍAZ ${ }^{2}$ \\ ${ }^{1}$ Hospital Pharmacy Department, Central University Hospital of Asturias, Oviedo, 33011 Asturias; \\ ${ }^{2}$ Faculty of Pharmacy, University of Granada, 18071 Granada, Spain
}

Received October 15, 2017; Accepted February 23, 2018

DOI: $10.3892 / \mathrm{ol} .2018 .8400$

\begin{abstract}
Sorafenib is an oral multikinase inhibitor with antiangiogenic and antiproliferative properties, and is used as the first-line treatment for patients with advanced hepatocellular carcinoma (HCC). Previous studies have identified an improvement in overall survival and progression-free survival in patients with a manageable toxicity profile. $\alpha$-fetoprotein (AFP) has been revealed to be of great diagnostic and predictive value for tumour staging in multiple studies; however, its role as a predictive factor of response to treatment with sorafenib is not entirely clear. The present study aimed to determine the effectiveness of sorafenib and investigate the value of AFP as a predictive factor of early response to sorafenib in patients with HCC. Effectiveness was analysed based on median overall survival (mOS) time, while to analyse the possible predictive value of AFP, patients were classified into two groups: Non-responders $(\leq 20 \%$ AFP reduction) and responders $(>20 \%$ AFP reduction) at 6-8 weeks of treatment when compared with basal AFP level. For assessment of toxicity, any adverse effects were recorded. A total of 167 patients were included, who collectively exhibited a mOS time of 11 months with a median treatment duration of 5 months. The mOS time was significantly higher for patients with better hepatic function (12 months in cases of Child-Pugh score A vs. 8 months in cases of Child-Pugh score B; $\mathrm{P}=0.03$ ) and with basal AFP values $\leq 200 \mathrm{ng} / \mathrm{ml}$ (14 months vs. 8 months in patients with AFP levels $>200 \mathrm{ng} / \mathrm{ml} ; \mathrm{P}=0.01)$. A $>20 \%$ reduction of AFP at 6-8 weeks was determined to be a positive predictive
\end{abstract}

Correspondence to: Miss Ana Isabel Plano Sánchez, Hospital Pharmacy Department, Central University Hospital of Asturias, Roma Avenue, Oviedo, 33011 Asturias, Spain

E-mail: planoana84@gmail.com

Key words: sorafenib, $\alpha$-fetoprotein, hepatocellular carcinoma, biomarker, effectiveness factor upon multivariate analysis $(\mathrm{P}=0.002)$, obtaining, for the responder patients, an mOS of 18 months compared with 10 months $(\mathrm{P}=0.004)$ for the non-responders. The main adverse reactions were hand-foot syndrome $(35 / 167 ; 21 \%)$, diarrhoea $(39 / 167 ; 23.4 \%)$, anorexia $(29 / 167 ; 17.4 \%)$ and arterial hypertension $(30 / 167 ; 18 \%)$. In conclusion, a $>20 \%$ drop in AFP at 6-8 weeks may be useful as a predictive factor of response to sorafenib, as indicated by its association with longer survival times in patients with advanced HCC following treatment with sorafenib in the present study.

\section{Introduction}

Hepatic cancer (HCC) is the sixth most common type of cancer, with $\sim 782,000$ new cancer cases in 2012 worldwide (1). In the majority of cases, HCC develops following cirrhosis of the liver, and is the primary cause of mortality in these patients $(1,2)$

The incidence of $\mathrm{HCC}$ has doubled in the last 20 years in the United States and Europe, and has exhibited the highest rate of increase among all cancer-associated causes of mortality in recent years (3). Approximately $90 \%$ of $\mathrm{HCC}$ cases are associated with known risk factors (4); the most frequent causes are chronic viral hepatitis [hepatitis C virus (HCV) and hepatitis B virus (HBV)], alcohol consumption, and exposure to aflatoxins (primarily in Africa and Asia), while other factors may also increase the risk, including obesity, tobacco use and fatty liver disease (5).

The most widely used technique in tumour staging is the Barcelona Clinic Liver Cancer (BCLC) system, as this is the only method that takes into account the spread of the tumour, hepatic function and the presence of symptoms, and thus has high predictive capacity (5). It establishes tumour prognosis in four stages, with each one having possible therapeutic indications. Patients with initial stage cancer (BCLC stage A) are candidates for potentially curative treatments, including surgical resection, percutaneous ablation and transplant, whereas those with intermediate stage (BCLC stage B) may be subject to palliative treatments, including arterial chemoembolization. In addition, sorafenib is used as a systematic treatment in the event of advanced HCC (BCLC stage C) (6). 
The prognosis of most solid tumours depends on the tumour stage; nevertheless, given that HCC is associated with hepatic cirrhosis in the majority of cases, and that the degree of hepatic function determines the therapeutic regimens and patient survival, assessing the level of hepatic dysfunction and tumour spreading is considered essential. Furthermore, the symptomatology has been shown to be of high predictive value, and is also indicative for selecting the most appropriate treatment.

With the current screening programmes performed, HCC is able to be diagnosed at earlier stages, thus making it possible to choose effective therapeutic methods. Surgical resection, transplant and percutaneous ablation achieve high complete response rates for tumours (7). Regarding palliative therapy, the only treatments to demonstrate an increase in survival rates have been chemoembolisation and sorafenib $(8,9)$. Other therapies, including arterial embolisation without chemotherapy, external radiotherapy and radioembolisation have been demonstrated to exhibit antitumour activity, but have not demonstrated efficacy in terms of survival $(10,11)$. Systematic chemotherapy with classic cytotoxic agents has not revealed any benefit for patient survival and is associated with high toxicity, and hormonal therapy, including the use of tamoxifen and octreotide, and antiandrogens, appears to be ineffective $(7,9)$. Sorafenib is an oral multikinase inhibitor with antiproliferative and antiangiogenic activity, which has demonstrated efficacy in improving the overall survival of patients with advanced HCC. It is currently the only first-line treatment indicated for advanced or intermediate-stage HCC following failure or contraindication of standard therapies (12).

In an international, multicentre, randomised and double-blind study (SHARP) (13), sorafenib (400 mg/12 h) was evaluated compared with placebo treatment in patients with advanced HCC exhibiting conserved hepatic function $(95 \%$ Child Pugh class A, 5\% Child Pugh class B) and practically asymptomatic conditions [Eastern Cooperative Oncology Group Performance (ECOG) score 0-2]. This study revealed a significant improvement in overall survival, as well as in median time-to-radiological progression (mOS) of 10.7 vs. 7.9 months in the placebo group.

A similarly designed clinical trial was performed in Asia (8), involving HCC cases at more advanced stages, with the large majority of cases involving tumours occurring secondary to cirrhosis caused by the HBV. Sorafenib exhibited benefits similar to those described in the SHARP study, with a reduction in the risk of mortality, although it achieved a lower median survival time (6.5 vs. 4.2 months in the placebo group).

The monitoring of responses to treatments involves the monitoring of patients by performing imaging tests, including computed tomography or magnetic resonance imaging, and measuring $\alpha$-fetoprotein (AFP) levels. In order to define the stage of the illness prior to and following treatment, it is recommended that the Response Evaluation Criteria In Solid Tumors (RECIST) criteria are used $(14,15)$. In general, clinical trials use imaging tests as a means of evaluating response to systematic treatments (14). However, numerous patients experience symptomatic improvement or evident improvement in pathology without exhibiting significant radiological changes (16). Numerous drugs exert cytostatic rather than cytotoxic activity, and inhibit the growth of tumours without causing a reduction in size (14). Furthermore, molecules, including sorafenib, induce tumour necrosis, which is occasionally associated with an increase in size or stabilisation of the tumour (14). The partial or complete response to treatment (assessed according to the RECIST criteria) is the primary independent predictive factor of survival in patients with HCC (14). Nonetheless, only in few cases is there a complete response to sorafenib, with a small proportion achieving a partial response (12). Furthermore, between 45 and $75 \%$ of patients exhibit stabilisation of the illness as their best response $(15,16)$. Thus, it is important to perform studies that assess possible biomarkers or measures of response, to allow us to determine probable response predictors (17).

AFP is a serum glycoprotein that is elevated in over half of patients with HCC (14). A number of studies have demonstrated its value at the beginning of diagnosis as a predictive factor of survival (18). Indeed, serum AFP level is used for diagnosis, as a predictive marker and to evaluate response following systematic chemotherapy or radiological therapies (15). A previous study of 25 patients treated with systematic chemotherapy revealed that a reduction in AFP level of $>50 \%$ was associated with a good clinical response to the treatment (19). Additionally, Chan et al (20) confirmed that a decline in basal AFP of $>20 \%$ following 2-3 cycles of systemic chemotherapy was predictive of response to treatment (20). However, only a small number of studies have been performed to investigate its role as a possible predictor of response following therapy with sorafenib in patients with HCC (21-23).

At present the use of sorafenib is indicated for patients with advanced HCC, for those who are not candidates for chemoembolization, or for those with post-chemoembolisation progression with conserved hepatic function (Child-Pugh class A).

The aim of the present study was to assess the effectiveness of sorafenib, and the value of AFP as a predictive factor of response, as well as other prognostic factors associated with overall survival, in patients diagnosed with advanced HCC treated with sorafenib as the first-line treatment, or in patients with advanced-stage disease following progression or resistance to standard treatment.

\section{Materials and methods}

Study design. The present study was an observational retrospective study of patients with HCC treated with sorafenib at the Central University Hospital of Asturias (Oviedo, Spain). The study was approved by the Ethical Research Committee of Central University Hospital of Asturias. Written informed consent was obtained for use of patient data, while maintaining patient anonymity and respecting patient confidentiality throughout.

\section{Study population}

Patients. Patients with HCC treated at the Central University Hospital of Asturias who started first-line treatment with sorafenib for advanced $\mathrm{C}$ stage, or as second-line treatment for intermediate stage $\mathrm{B}$ due to resistance to standard treatment 
or progression, were enrolled. During the study period, 167 patients began treatment with sorafenib with a median age of 65.6 years (range, 23.9-80.6 years), and 147 (88\%) men and $20(12 \%)$ women.

Inclusion criteria. All patients diagnosed with HCC who started treatment with sorafenib between January 2008 and December 2014 were included. The end date of the monitoring period was the 20th of December 2015.

Exclusion criteria. All those being treated with sorafenib during a clinical trial, along with those who had begun treatment prior to 2008, when access to the drug was granted on request for compassionate use only were excluded. Those patients who lacked records for the variables studied on the computer systems used were also excluded, unless otherwise stated.

Treatments. All patients received $800 \mathrm{mg} /$ day sorafenib (400 mg twice/day) as an initial dosage. Dosage reductions took place in accordance with the technical file upon signs of toxicity or adverse effects. The patients continued treatment unless there was clinical progression, unacceptable toxicity, or cases of patient refusal or patient mortality.

\section{Variable definitions}

Main variables. To assess the effectiveness of the treatment, the median overall survival (mOS) time, calculated as the interval (in days) between the beginning of treatment and the date of mortality, if mortality occurred, or the end date of monitoring for patients who survived (20th of December 2015) were used. To evaluate the possible predictive value of AFP in response to treatment, the patients were classified into two groups: Those with AFP reduction $\leq 20 \%$ (non-responders) and those with AFP reduction $>20 \%$ (responders), measured at 6-8 weeks after starting treatment with sorafenib.

Secondary variables. To assess other possible factors predictive of survival, the ECOG score $(0,1$ or 2$)$, aetiology, stage according to the BCLC system (BCLC stage B or C) and hepatic function based on the Child-Pugh classification (A or B) for each patient were determined. Additionally, whether or not at the beginning of the treatment the patient presented vascular invasion and/or extrahepatic extension was recorded. The analytical parameters recorded were basal serum levels of albumin ( $>35$ or $\leq 35 \mathrm{~g} / \mathrm{l})$, basal serum AFP ( $>200$ or $\leq 200 \mathrm{ng} / \mathrm{dl}$ ) and serum AFP at 6-8 weeks. Any other treatments administered prior to the beginning of sorafenib treatment were also recorded.

Toxicity. The adverse effects and the duration of the treatment were recorded. Any suspensions of treatment due to toxicity or an unknown factor, including low patient compliance, were also recorded.

Statistical analysis. A descriptive statistical analysis of the demographic and clinical variables was performed using centralisation and dispersion methods (median and interquartile range) for the quantitative data, and frequencies and proportions for the qualitative data. Duration of the treatment was determined (in months), along with the time of appearance of any adverse affects. In order to determine overall survival, Kaplan-Meier curves were constructed and the differences between groups were analysed via the log-rank test. To identify the possible predictive factors associated with overall survival, the Cox proportional hazards model was used to determine respective hazard quotients and $95 \%$ confidence intervals (CIs). The variables identified as potential predictors of effectiveness in the univariate analysis were subsequently included in the multivariate analysis. For all of the analyses, $\mathrm{P}<0.05$ was considered to indicate a statistically significant difference. Statistical analysis was performed using the SPSS statistical data package (version 19.0; IBM Corp., Armonk, NY, USA).

\section{Results}

Patient characteristics. All of the patients were diagnosed with advanced HCC; $82.3 \%$ of cases also presented with a cirrhosis of the liver and $61.7 \%$ exhibited advanced-stage HCC (BCLC stage C). Alcohol consumption was the primary cause of HCC development $(40.1 \%)$, followed by chronic $\mathrm{HCV}$ infection (21.6\%). At the start of treatment, the majority of patients had preserved hepatic function, with Child-Pugh class A (42.5\% patients). Clinical data regarding the spread of the disease was identified as incorrectly defined in certain registries, and therefore it was only possible to analyse a percentage of the total number of patients for this variable. Of the 117 patients for whom the presence or absence of extrahepatic extension was documented, this was positive in 46 cases ( $27.5 \%$ of the total). Information on vascular invasion was only assessable for 130 patients, with 80 cases (47.9\% of the total) being positive for vascular invasion. Regarding basal AFP serum levels, 49 (29.3\%) patients presented values $>200 \mathrm{ng} / \mathrm{ml}$. The demographical and clinical characteristics of patients are outlined in Table I.

Treatment. Prior to beginning treatment with sorafenib, 53 $(31.7 \%)$ patients had not received any treatment, $64(38.3 \%)$ patients had received locoregional therapy [transarterial chemoembolization (TACE) and/or radiofrequency ablation], $18(10.8 \%)$ had undergone surgical resection with curative intent prior to sorafenib treatment and 7 (4.2\%) had received liver transplants. No information was available on previous treatments for 25 patients.

The median duration of treatment was 5 months. During monitoring, $92(55.1 \%)$ patients required a reduction in dosage. Progression of the illness was the primary reason for ending treatment in $94(56.3 \%)$ patients (including those who succumbed while being treated), followed by adverse effects (41 patients, 24.6\%). A total of 2 patients were moved on to trials with tivantinib, $2(1.1 \%)$ with regorafenib, 1 with nivolumab $(0.5 \%)$ and another to treatment with tamoxifen $(0.5 \%)$, following the suspension of sorafenib.

Effectiveness and survival in different subgroups. On analysis of overall survival, an mOS time of 11 months was determined (95\% CI, 8.65-13.34). Two patients exhibited complete responses for 17 and 24 months, respectively, with both remaining alive until the date the monitoring ended. 
Table I. Baseline demographical and clinical characteristics of patients.

\begin{tabular}{lc}
\hline Variable & Value \\
\hline Total patients, $\mathrm{n}$ & 167 \\
Age, years [median (range)] & 65.6 \\
& $(23.9-80.6)$ \\
Sex, n (\%) & \\
Male & $147(88)$ \\
Female & $20(12)$
\end{tabular}

Child-Pugh class, n (\%)

A

B

ECOG performance status, $\mathrm{n}(\%)$

0

1

2

Aetiology of HCC, n (\%)

VHB cirrhosis

$\mathrm{VHC}$ cirrhosis

VHC cirrhosis and alcohol

VHB cirrhosis and alcohol

Alcoholic cirrhosis

Hepatitis C+HIV

Hemochromatosis

$\mathrm{HCV}+\mathrm{HBV}$

Other

29 (17.4)

4 (2.4)

5 (3)

36 (21.6)

$17(10.2)$

4 (2.4)

$67(40.1)$

5 (3)

$5(3)$

$1(0.6)$

27 (16.1)

BCLC stage, n (\%)

$\mathrm{B}$

38 (22.8)

$103(61.7)$

$80(47.9)$

$46(27.5)$

7 (4.2)

76.6

(1.2-233800.0)

49 (29.3)

$118(70.7)$

Basal AFP $\leq 200 \mathrm{ng} / \mathrm{ml}, \mathrm{n}(\%)$

Basal hepatic enzymes, IU/1 [median (range)]

Aspartate aminotransferase

$58(19-344)$

Alanine aminotransferase

$44.5(13-274)$

Basal albumin value, g/l [median (range)]

$39(25-48)$

$63(37.7)$

$104(62.3)$

Basal albumin >35 g/l, n (\%)

$1(0.2-4.4)$

Basal bilirubin value, $\mathrm{mg} / \mathrm{ml}$ [median (range)]

Basal bilirubin $\leq 1 \mathrm{mg} / \mathrm{ml}, \mathrm{n}(\%)$

80 (47.9)

$87(52.1)$

Basal bilirubin $>1 \mathrm{mg} / \mathrm{ml}, \mathrm{n}(\%)$

AFP reduction at 6-8 weeks, $\mathrm{n}(\%)$

AFP responders $(>20 \%)$

$28(16.8)$

AFP non-responders $(\leq 20 \%)$

Certain data were not available for all patients. AFP, $\alpha$-fetoprotein; BCLC, Barcelona Clinic Liver Disease; ECOG, Eastern Cooperative Oncology Group Performance; HCV, hepatitis C virus; HBV, hepatitis B virus; HIV, human immunodeficiency virus.

Statistical analyses of survival was performed between subgroups based on potential risk factors, including age, Child Pugh classification, BCLC stage, ECOG score, basal AFP $>200 \mathrm{ng} / \mathrm{ml}$ and AFP reduction at 6-8 weeks.

With regards to the basal AFP levels, notable differences in survival were observed, in that mOS time was significantly lower in those with initial AFP levels $>200 \mathrm{ng} / \mathrm{ml}$ (mOS, 8 months vs. 14 months in patients with AFP levels $\leq 200 \mathrm{ng} / \mathrm{ml})$ $(\mathrm{P}=0.01$; Fig. 1).

Another clinical variable that was associated with significantly higher mOS was ECOG performance status, with an mOS of 16 months identified for patients with an ECOG of 0 , compared with an mOS of 4 months in patients with an ECOG of $2(\mathrm{P}=0.001)$. Hepatic function was also an influential factor, with Child-Pugh class A associated with a significantly higher mOS of 12 months, compared with 8 months for Child-Pugh class $\mathrm{B}$ ( $\mathrm{P}=0.03$; Fig. 2). Lastly, those patients aged $>63$ years exhibited a higher mOS time (14 months vs. 8 months in patients $\leq 63$ years old; $\mathrm{P}=0.03$ ). Patients at $\mathrm{BCLC}$ stage $\mathrm{B}$ exhibited increased mOS times compared with patients at BCLC stage C (15 vs. 10 months), without reaching statistical significance.

Role of AFP reduction as a predictive factor of early response. Of the 167 patients, 28 belonged to the AFP responders group, whereas 139 belonged to the non-responder group. The AFP responder patients exhibited a difference in mOS compared with the non-responders (18 vs. 10 months; Fig. 3; $\mathrm{P}=0.004)$.

A multivariate analysis was performed to assess the potency of the AFP variable as an independent predictive factor, as well as the value of the other clinical variables that were significant in the univariate analysis (Table II). A reduction in AFP by $>20 \%$ at 6-8 weeks was an independent factor associated with higher mOS time $(\mathrm{P}=0.002)$.

Basal AFP levels of $\leq 200 \mathrm{ng} / \mathrm{ml}$ and age $>63$ years were also identified as independent factors associated with higher mOS time.

Toxicity. With regards to the toxicity of treatment, the primary adverse reactions registered in the medical records were hand-foot syndrome (35 patients, 21\%), diarrhoea (39 patients, 23.4\%), anorexia (29 patients, 17.4\%) and arterial hypertension (30 patients, 18\%). Seven cases of encephalopathy were registered $(4.2 \%), 1$ patient suffered an ischaemic stroke $(0.6 \%), 2$ exhibited angina with atrial fibrillation $(1.2 \%), 1$ exhibited myocardial infarction $(0.6 \%)$ and treatment was suspended for 2 patients $(1.2 \%)$ due to intense haemorrhoid bleeding.

\section{Discussion}

A number of studies have demonstrated that sorafenib is effective in the treatment of HCC, particularly by increasing the overall survival times compared with placebos, while having a manageable toxicity profile $(13,24,25)$. Thus, it has become established as a first-line treatment for patients with advanced HCC. In clinical practice, serum AFP is used as a biomarker in HCC. There are studies that support the efficacy of AFP level as a predictive factor and as a response factor following surgical resection $(16,26)$; nonetheless, its 


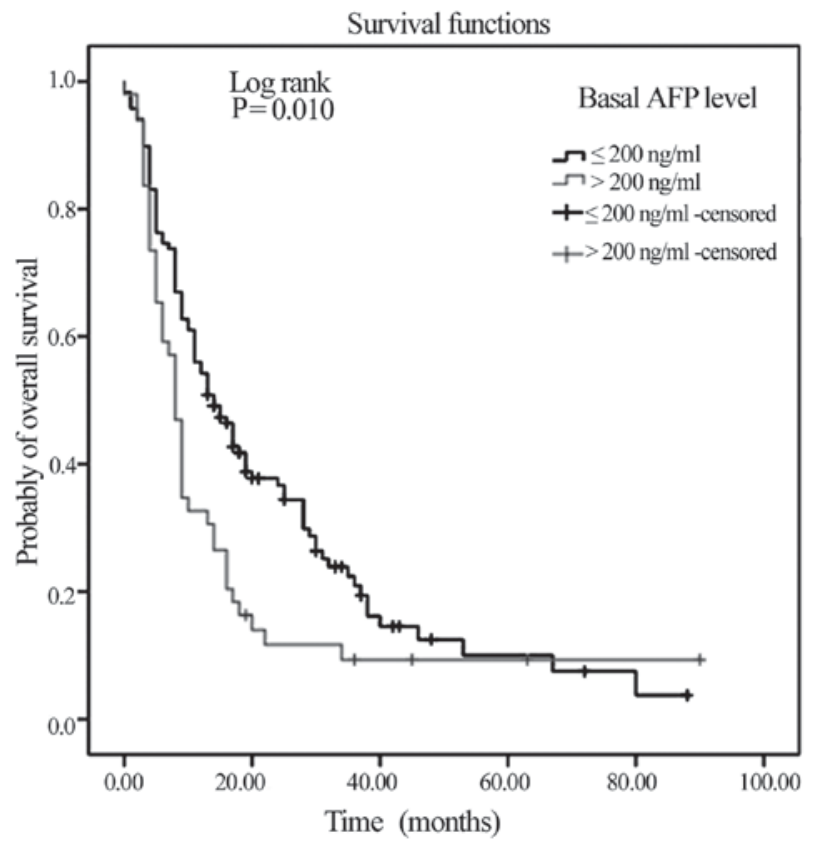

Figure 1. Kaplan-Meier Curve of overall survival in the $>200$ and $\leq 200 \mathrm{ng} / \mathrm{ml}$ basal AFP risk subgroups. AFP, $\alpha$-fetoprotein.

usefulness as a predictive factor of response to treatment with sorafenib or other anti-angiogenics drugs is not clearly established.

The results regarding the overall survival and toxicity profile associated with sorafenib in the present study are similar to those reported in the SHARP trial (13). This may be due to similarities in the demographical and clinical characteristics collected from the patients in the present study and those in the SHARP study (13), which reported a higher incidence in males $(88 \%)$ and in those aged $\sim 65$ years (median, 65.6 years), involving patients in a good general state $(92 \%$, ECOG score 0-1) and with advanced-stage HCC (73\%).

Regarding prior treatments, nearly half of the patients $(71 / 167,42.5 \%)$ in the current study had been treated with other optional palliative therapies, with a predominance of TACE at a similar frequency to that in the SHARP trial, with the only difference being that no patient in the present study had previously received hormone therapy or systematic chemotherapy. A sub-analysis of the SHARP trial performed by Bruix et al (24) identified a tendency towards improvement in overall survival in patients who received prior therapies independently of the treatment received, with an mOS time of 8.8 months for those treated with curative therapy and 9.9 months for those who received other palliative treatments. Indeed, in the current study cohort, a difference in mOS time (24) between patients treated with curative therapy (25 months) and those who had received other palliative treatments was observed (11 months; $95 \% \mathrm{CI}$, 8.3-41.6 and 7.8-14.1, respectively).

The present study cohort exhibited an overall mOS time (11 months) similar to the SHARP trial (10.7 months) and slightly lower compared with that in the study by Kudo et al (26) (13.7 months). However, it was higher compared with that in an extended population of the SHARP study involving an Asian population (6.5 months), (8) as well as in other subsequent

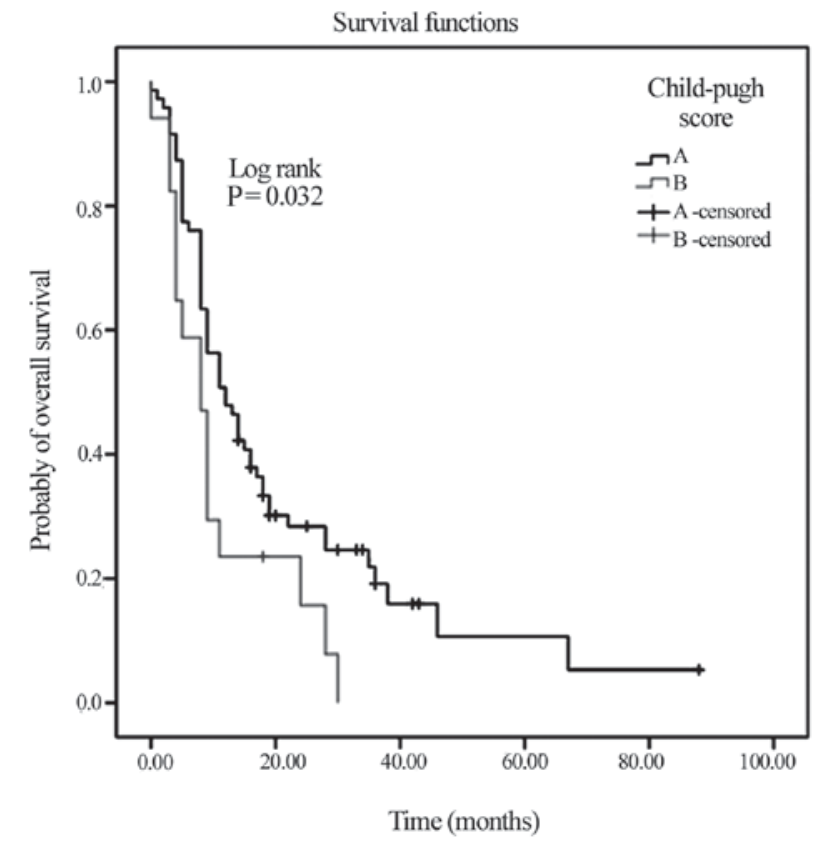

Figure 2. Kaplan-Meier Curve of overall survival in the Child-Pugh class A and $\mathrm{B}$ risk subgroups.

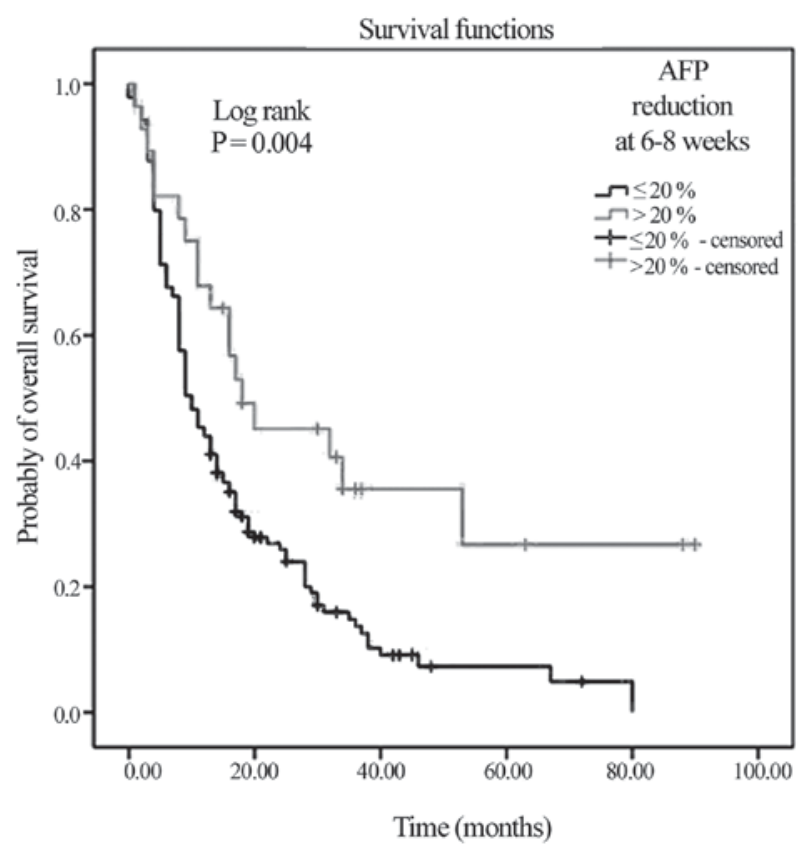

Figure 3. Kaplan-Meier Curve of overall survival based on AFP reduction in the non-responder ( $\leq 20 \%$ AFP reduction) and responder $(>20 \%$ AFP reduction) risk subgroups. AFP, $\alpha$-fetoprotein.

studies performed (5.4 months) (27). Previous data exhibit a tendency towards improvement in survival for those patients with good liver function and general state (ECOG performance status) (28-30). Regarding the parameters analysed, high levels of basal AFP were an indicator of poor prognosis in the present study, and have previously been associated with shorter survival times (27,31). A sub-analysis of the SHARP trial performed by Raoul et al (32) analysed the influence of laboratory parameters, including hepatic enzymes, AFP and bilirubin, on the response to treatment with sorafenib. 
Table II. Univariate and multivariate analysis for the possible predictive factors of OS.

\begin{tabular}{|c|c|c|c|c|}
\hline \multirow[b]{2}{*}{ Factor } & \multicolumn{2}{|c|}{ Univariate analysis } & \multicolumn{2}{|c|}{ Multivariate analysis } \\
\hline & HR $(95 \% \mathrm{CI})$ & P-value & $\operatorname{HR}(95 \% \mathrm{CI})$ & P-value \\
\hline Age (>63 vs. $\leq 63$ years $)$ & $1.433(1.015-2.023)$ & 0.041 & $3.895(1.727-8.783)$ & 0.001 \\
\hline ECOG performance status (0 vs. 1) & $1.709(1.050-2.781)$ & 0.006 & $1.120(0.562-2.233)$ & 0.697 \\
\hline ECOG performance status (0 vs. 2) & $4.137(0.462-11.707)$ & 0.007 & $1.913(0.397-9.206)$ & 0.419 \\
\hline Child-Pugh class (A vs. B) & $1.787(1.018-3.138)$ & 0.043 & $0.413(0.157-1.089)$ & 0.074 \\
\hline BCLC stage (B vs. C) & $1.290(0.850-1.958)$ & 0.231 & & \\
\hline Basal AFP ( $\leq 200$ vs. $>200$ ng/ml) & $1.583(1.103-2.272)$ & 0.013 & $2.694(0.392-5.212)$ & 0.003 \\
\hline $\begin{array}{l}\text { AFP reduction }[\text { Responders }(>20 \%) \text { vs. } \\
\text { Non-responders }(\leq 20)]\end{array}$ & $2.032(1.230-3.357)$ & 0.006 & $9.723(2.252-41.969)$ & 0.002 \\
\hline
\end{tabular}

Patients who demonstrated elevated levels of these markers had OS rate shorter (although the sorafenib cohort had greater OS rate, compared with the placebo group), compared with patients whose markers were normalised in both cohorts (32). Thus, the authors concluded that these markers had predictive value in $\mathrm{HCC}$, but were not predictive of response to treatment with sorafenib in the HCC patients. In the present study, when analysing mOS time according to subgroups, the patients who presented AFP values of $>200 \mathrm{ng} / \mathrm{ml}$ exhibited a significantly lower mOS time, compared with the AFP $\leq 200 \mathrm{ng} / \mathrm{ml}$ group. Another study performed on a Korean population by Lee et al (33) revealed that liver function (Child-Pugh class A) and low basal AFP levels were independent risk factors associated with longer mOS times $(\mathrm{P}=0.02$ and $\mathrm{P}=0.03$, respectively) (33). In the current group of patients, AFP levels $>200 \mathrm{ng} / \mathrm{ml}$ was identified as an independent predictor of shorter survival time in the multivariate analysis; nevertheless, good liver function (Child-Pugh class A), despite indicating improved survival (12 vs. 8 months in cases of Child Pugh class B) in the subgroup analysis ( $\mathrm{P}>0.05)$, was not deemed to be statistically significant as an independent predictive factor on performance of the multivariate test.

A number of studies performed with different systemic chemotherapy treatments determined that a drop in AFP level may be useful as a predictive marker for tumour response and overall survival $(17,20,34)$, although no consensus was achieved regarding the amount of reduction and the measurement times. Personeni et al (21) classified those patients with a reduction in AFP levels of $\geq 20 \%$ at $6-8$ weeks as responders, and those with an AFP of $\leq 20 \%$ as non-responders. Another study restricted the definition of responder to a drop in AFP of $>50 \%$, thus increasing the potency and specificity of AFP as a predictor of response treatment (35). A reduction in AFP measured at 6-8 weeks is considered relevant and applicable in clinical practice for aggressive pathologies, including $\mathrm{HCC}$, with short mOS times of 4-6 months, with treatments that achieve this also having a beneficial impact on medical costs $(8,27)$. This study demonstrated that an early drop in AFP level (>20\%) by $6-8$ weeks of treatment with sorafenib is associated with improved patient survival, with higher greater mOS time observed for the AFP responder group (18 vs. 10 months in the non-responder group, log rank $\mathrm{P}=0.004)$, which is in accordance with other previous studies $(22,36)$, despite a number of these not reaching statistical relevance $(28,37)$.

In addition, a $>20 \%$ reduction in AFP was a significant independent predictive factor of response to treatment in the univariate analysis and subsequent multivariate analysis. Thus, $>20 \%$ AFP reduction was useful as a predictive marker of early response to sorafenib in patients with HCC, being associated with a higher survival rate. This quickly detectable biomarker requiring inexpensive methods may predict whether treatment is effective, and ultimately identify patients who may benefit from a continuation of treatment from those who should be referred for alternative therapies.

Although high levels of AFP are associated with negative prognoses, the value of AFP as an early response marker to sorafenib in patients with HCC is not entirely clear. It is necessary to perform prospective studies specifically designed to assess the potency of AFP reduction in predicting patient prognosis to validate the preliminary results of the present study. Additionally, the current study included a number of limitations, primarily deriving from its retrospective design: Data were not available for all variables for the 167 patients, and were included as missing in the statistical analysis, which thus resulted in a loss of potency.

\section{Acknowledgements}

The authors thank Sabina Pérez Vicente (Results Evaluation Unit, Institute of Biomedicine of Sevilla) for her help with the statistical analysis.

\section{Funding}

No funding was received.

\section{Availability of data and materials}

The datasets used and/or analyzed during the current study are available from the corresponding author on reasonable request. 


\section{Authors' contributions}

AIPS conceived and designed the study, performed acquisition, analysis and interpretation of data, and wrote the manuscript. LVR assisted with the design of the study. ELL participated in the data compilation. IZG reviewed the manuscript and interpreted the data. MACH helped to design the methods of the study. MIBP and JPD helped to interpret the data and reviewed critically the intellectual content of the manuscript. All authors read and approved the final manuscript

\section{Ethics approval and consent to participate}

The study was approved by the Ethical Research Committee of Central University Hospital of Asturias. Written informed consent was obtained for use of patient data, while maintaining patient anonymity and respecting patient confidentiality throughout.

\section{Consent for publication}

All identifying information has been removed.

\section{Competing interests}

The authors declare that they have no competing interests.

\section{References}

1. International Agency for Research on Cancer (IARC): Globocan 2012: Estimated Cancer Incidence, Mortality and Prevalence Worldwide in 2012. http://globocan.iarc.fr/Default. aspx. Accessed April 10, 2015.

2. Forner A, Llovet JM and Bruix J: Hepatocellular carcinoma. Lancet 379: 1245-1255, 2012.

3. Mittal S and El-Serag HB: Epidemiology of hepatocellular carcinoma: Consider the population. J Clin Gastroenterol 47: S2-S6, 2013.

4. European Association for the Study of The Liver; European Organisation for Research and Treatment of Cancer: EASL-EORTC clinical practice guidelines: Management of hepatocellular carcinoma. J Hepatol 56: 908-943, 2012.

5. Omata M, Cheng AL, Kokudo N, Kudo M, Lee JM, Jia J, Tateishi R, Han KH, Chawla YK, Shiina S, et al: Asia-pacific clinical practice guidelines on the management of hepatocellular carcinoma: A 2017 update. Hepatol Int 11: 317-370, 2017

6. Reig M, Matilla A, Bustamante J, Castells L, de La Mata M, Delgado M, Moreno JM, Forner A and Varela M: Recommendations for the management of Sorafenib in patients with hepatocellular carcinoma. Gastroenterol Hepatol 33: 741-752, 2010 (In Spanish).

7. Bruix J and Sherman M; American Association for the Study of Liver Diseases: Management of hepatocellular carcinoma: An update. Hepatology 53: 1020-1022, 2011.

8. Cheng AL, Kang YK, Chen Z, Tsao CJ, Qin S, Kim JS, Luo R, Feng J, Ye S, Yang TS, et al: Efficacy and safety of sorafenib in patients in the asia-pacific region with advanced hepatocellular carcinoma: A phase III randomised, double-blind, placebo-controlled trial. Lancet Oncol 10: 25-34, 2009.

9. Llovet JM and Bruix J: Systematic review of randomized trials for unresectable hepatocellular carcinoma: Chemoembolization improves survival. Hepatology 37: 429-442, 2003.

10. Dawson LA: The evolving role of radiation therapy in hepatocellular carcinoma. Cancer Radiother 12: 96-101, 2008.

11. Han KH, Seong J, Kim JK, Ahn SH, Lee DY and Chon CY: Pilot clinical trial of localized concurrent chemoradiation therapy for locally advanced hepatocellular carcinoma with portal vein thrombosis. Cancer 113: 995-1003, 2008

12. Colagrande S, Regini F, Taliani GG, Nardi C and Inghilesi AL: Advanced hepatocellular carcinoma and sorafenib: Diagnosis, indications, clinical and radiological follow-up. World J Hepatol 7: 1041-1053, 2015.
13. Llovet JM, Ricci S, Mazzaferro V, Hilgard P, Gane E, Blanc JF, de Oliveira AC, Santoro A, Raoul JL, Forner A, et al: Sorafenib in advanced hepatocellular carcinoma. N Engl J Med 359: 378-390, 2008 .

14. Lencioni R and Llovet JM: Modified recist (mRECIST) assessment for hepatocellular carcinoma. Semin Liver Dis 30: 52-60, 2010.

15. Kawaoka T, Aikata H, Murakami E, Nakahara T, Naeshiro N, Tanaka M, Honda Y, Miyaki D, Nagaoki Y, Takaki S, et al: Evaluation of the mrecist and $\alpha$-fetoprotein ratio for stratification of the prognosis of advanced-hepatocellular-carcinoma patients treated with sorafenib. Oncology 83: 192-200, 2012.

16. Takada J,Hidaka H, Nakazawa T, Kondo M, Numata K, Tanaka K, Matsunaga K, Okuse C, Kobayashi S, Morimoto M, et al: Modified response evaluation criteria in solid tumors is superior to response evaluation criteria in solid tumors for assessment of responses to sorafenib in patients with advanced hepatocellular carcinoma. BMC Res Notes 8: 609, 2015.

17. Yau T, Yao TJ, Chan P, Wong H, Pang R, Fan ST and Poon RT: The significance of early alpha-fetoprotein level changes in predicting clinical and survival benefits in advanced hepatocellular carcinoma patients receiving sorafenib. Oncologist 16: 1270-1279, 2011

18. Nomura F, Ohnishi K and Tanabe Y: Clinical features and prognosis of hepatocellular carcinoma with reference to serum alpha-fetoprotein levels. Analysis of 606 patients. Cancer 64: 1700-1707, 1989.

19. Matsumoto Y, Suzuki T, Ono H, Nakase A and Honjo I: Evaluation of hepatoma chemotherapy by alpha-fetoprotein determination. Am J Surg 132: 325-328, 1976.

20. Chan SL, Mo FK, Johnson PJ, Hui EP, Ma BB, Ho WM, Lam KC, Chan AT, Mok TS and Yeo W: New utility of an old marker: Serial alpha-fetoprotein measurement in predicting radiologic response and survival of patients with hepatocellular carcinoma undergoing systemic chemotherapy. J Clin Oncol 27: 446-452, 2009.

21. Personeni N, Bozzarelli S, Pressiani T, Rimassa L, Tronconi MC, Sclafani F, Carnaghi C, Pedicini V, Giordano L and Santoro A: Usefulness of alpha-fetoprotein response in patients treated with sorafenib for advanced hepatocellular carcinoma. J Hepatol 57: 101-107, 2012.

22. Liu L, Zhao Y, Jia J, Chen H, Bai W, Yang M, Yin Z, He C, Zhang L, Guo W, et al: The prognostic value of alpha-fetoprotein response for advanced-stage hepatocellular carcinoma treated with sorafenib combined with transarterial chemoembolization. Sci Rep 6: 19851, 2016.

23. Okuyama H, Ikeda M, Kuwahara A, Takahashi H, Ohno I, Shimizu S, Mitsunaga S, Senda S and Okusaka T: Prognostic factors in patients with hepatocellular carcinoma refractory or intolerant to sorafenib. Oncology 88: 241-246, 2015.

24. Bruix J, Raoul JL, Sherman M, Mazzaferro V, Bolondi L, Craxi A, Galle PR, Santoro A, Beaugrand M, Sangiovanni A, et al: Efficacy and safety of sorafenib in patients with advanced hepatocellular carcinoma: Subanalyses of a phase III trial. J Hepatol 57: 821-829, 2012.

25. Iavarone M, Cabibbo G, Piscaglia F, Zavaglia C, Grieco A, Villa $\mathrm{E}, \mathrm{Cammà} \mathrm{C}$, Colombo $\mathrm{M}$ and SOFIA (SOraFenib Italian Assessment) study group: Field-practice study of sorafenib therapy for hepatocellular carcinoma: A prospective multicenter study in Italy. Hepatology 54: 2055-2063, 2011.

26. Kudo M, Imanaka K, Chida N, Nakachi K, Tak WY, Takayama T, Yoon JH, Hori T, Kumada H, Hayashi N, et al: Phase III study of sorafenib after transarterial chemoembolisation in Japanese and Korean patients with unresectable hepatocellular carcinoma. Eur J Cancer 47: 2117-2127, 2011.

27. Køstner AH, Sorensen M, Olesen RK, Grønbæk H, Lassen U and Ladekarl M: Sorafenib in advanced hepatocellular carcinoma: A nationwide retrospective study of efficacy and tolerability. ScientificWorldJournal 2013: 931972, 2013.

28. DA Fonseca LG, Barroso-Sousa R, Bento AD, Blanco BP, Valente GL, Pfiffer TE, Hoff PM and Sabbaga J: Safety and efficacy of sorafenib in patients with Child-Pugh B advanced hepatocellular carcinoma. Mol Clin Oncol 3: 793-796, 2015.

29. Nakano M, Tanaka M, Kuromatsu R, Nagamatsu H, Tajiri N, Satani M, Niizeki T, Aino H, Okamura S, Iwamoto H, et al: Kurume liver cancer study group of japan. sorafenib for the treatment of advanced hepatocellular carcinoma with extrahepatic metastasis: A prospective multicenter cohort study. Cancer Med 4: 1836-1843, 2015. 
30. Wang Z, Wu XL, Zeng WZ, Xu GS, Xu H, Weng M, Hou JN and Jiang MD: Meta-analysis of the efficacy of sorafenib for hepatocellular carcinoma. Asian Pac J Cancer Prev 14: 691-694, 2013.

31. Shao YY, Lin ZZ, Hsu C, Shen YC, Hsu CH and Cheng AL: Early alpha-fetoprotein response predicts treatment efficacy of antiangiogenic systemic therapy in patients with advanced hepatocellular carcinoma. Cancer 116: 4590-4596, 2010.

32. Raoul JL, Bruix J, Greten TF, Sherman M, Mazzaferro V, Hilgard P, Scherubl H, Scheulen ME, Germanidis G, Dominguez S, et al: Relationship between baseline hepatic status and outcome, and effect of sorafenib on liver function: SHARP trial subanalyses. J Hepatol 56: 1080-1088, 2012.

33. Lee S, Kim BK, Kim SU, Park SY, Kim JK, Lee HW, Park JY, Kim DY, Ahn SH, Tak WY, et al: Clinical outcomes and prognostic factors of patients with advanced hepatocellular carcinoma treated with sorafenib as first-line therapy: A korean multicenter study. J Gastroenterol Hepatol 29: 1463-1469, 2014.

34. Chen LT, Liu TW, Chao Y, Shiah HS, Chang JY, Juang SH, Chen SC, Chuang TR, Chin YH and Whang-Peng J: Alpha-fetoprotein response predicts survival benefits of thalidomide in advanced hepatocellular carcinoma. Aliment Pharmacol Ther 22: 217-226, 2005
35. Raoul JL, Park JW, Kang YK, Finn RS, Kim JS, Yeo W, Polite BN, Chao Y, Walters I, Baudelet C and Lencioni R: Using modified recist and alpha-fetoprotein levels to assess treatment benefit in hepatocellular carcinoma. Liver Cancer 3: 439-450, 2014.

36. Chou WC, Lee CL, Yang TS, Huang CY, Teng W, Tseng YT, Chen JS, Lin YC, Hou MM, Chang HH and Chia-Hsun Hsieh J: Changes in serum $\alpha$-fetoprotein level predicts treatment response and survival in hepatocellular carcinoma patients and literature review. J Formos Med Assoc 117: 153-163, 2018.

37. Xu XS, Qu K, Liu C, Zhang YL, Liu J, Song YZ, Zhang P, Liu SN and Chang HL: Highlights for $\alpha$-fetoprotein in determining prognosis and treatment monitoring for hepatocellular carcinoma. World J Gastroenterol 18: 7242-7250, 2012. 